\title{
Effects of hydration and temperature on side-chain conformational heterogeneity in protein crystals
}

\author{
Authors: Hakan Atakisi, David W. Moreau and Robert E. Thorne \\ Cornell University
}

Keywords: protein crystallography, protein structure, crystal dehydration, conformational heterogeneity, lysozyme, variable-temperature crystallography, protein-solvent interactions

Protein crystals contain large amounts of solvent, typically $30-70 \%$ by volume, that fills intermolecular spaces within the unit cell as well as internal protein cavities. Crystal solvent content depends on relative humidity (r.h.). Monoclinic lysozyme provides a remarkable model for studying structural changes induced by dehydration, as it maintains excellent order for relative humidities down to $5 \%$. In this study, the modulation of main-chain and side-chain conformational heterogeneity and solvent structure in monoclinic lysozyme crystals by dehydration and temperature is examined.

Decreasing the relative humidity (from 99\% to 11\%) and decreasing the temperature both lead to contraction of the unit cell, to an increased area of crystal contacts and to remodeling of primarily contact and solvent-exposed residues. Both lead to the depopulation of some minor side-chain conformers and to the generation of new conformations. Dehydration from $99 \%$ to $93 \%$ r.h. and cooling from 300 to $100 \mathrm{~K}$ result in a comparable number of remodeled residues, with dehydration-induced remodeling somewhat more likely to arise from contact interactions. When scaled to equivalent temperatures based on unit-cell contraction, the evolution of sidechain order parameters with dehydration shows generally similar features to those observed on cooling to $\mathrm{T}=100 \mathrm{~K}$.

These results illuminate the qualitative and quantitative similarities between structural perturbations induced by modest dehydration, which routinely occurs in samples prepared for 298 and $100 \mathrm{~K}$ data collection, and cryocooling. Differences between these perturbations in terms of energy landscapes and occupancies, and implications for variable-temperature crystallography between 180 and $300 \mathrm{~K}$, are discussed. 